\title{
DISERTACIONES
}

ENSAYOS

Anuario electrónico de estudios en Comunicación Social

ISSN: $1856-9536$

Doi: dx.doi.org/10.12804/disertaciones.09.02.2016.03

Volumen 9, Número 2 / julio-diciembre 2016

Versión PDF para imprimir desde

http://revistas.urosario.edu.co/index.php/disertaciones

Para citar este artículo: Hamelink, C. y Nordenstreng, K. (2016). Estudiando la historia a través de la Asociación Internacional para el Estudio de la Comunicación Social (IAMCR). Anuario Electrónico de Estudios en Comunicación Social "Disertaciones", 9(2), 46-67. Doi: dx.doi.org/10.12804/disertaciones.09.02.2016.03

\section{ESTUDIANDO LA HISTORIA A TRAVÉS DE LA ASOCIACIÓN INTERNACIONAL PARA EL ESTUDIO DE LA COMUNICACIÓN SOCIAL (IAMCR)}

Looking at History Through the International Association for Media and Communication Research (IAMCR) ${ }^{*}$

Estudando a história através da Associação Internacional para o Estudo da Comunicação Social (IAMCR)

Hamelink, Cees. Universiteit van Amsterdam (Países Bajos)

cjhamelink@gmail.com

Nordenstreng, Kaarle. University of Tampere (Finlandia)

kaarle.nordenstreng@uta.fi

Traducido por Miguel Vicente Mariño, Universidad de Valladolid (España)

* Este ensayo está basado en un proyecto conjunto entre los dos autores sobre la historia de la IAMCR. Sus primeros resultados fueron un folleto publicado con motivo del $50^{\text {a }}$ conferencia de la asociación, IAMCR in Retrospect (Hamelink y Nordenstreng, 2007), una entrada IAMCR en la International Encyclopedia of Communication (Hamelink, 2008) y el capítulo Institutional networking: The story of the International Association for Media and Communication Research (IAMCR)"en el libro The History of Media and Communication Research: Contested Memories (Nordenstreng, 2008). Este proyecto culminará con un libro sobre la historia de la IAMCR en el contexto de la historia del campo. 


\title{
DISERTACIONES
}

ENSAYOS

Anuario electrónico de estudios en Comunicación Social

ISSN: 1856-9536

Doi: dx.doi.org/10.12804/disertaciones.09.02.2016.03

Volumen 9, Número 2 / julio-diciembre 2016

Versión PDF para imprimir desde

Fecha de recibido: 22 de febrero de 2016

Fecha de aceptado: 29 de marzo de 2016

\section{RESUMEN}

Las historias del campo emergente de la comunicación de masas (Hardt, 2001; Pietilä, 2005) -en Europa continental desde finales del siglo xvı en adelante y en Estados Unidos desde comienzos del siglo xix en adelante- nos permiten reconocer el escaso y tardío rol que han jugado las instituciones internacionales en moldear la investigación en comunicación. A pesar de que las raíces del campo remiten a los clásicos de la sociología y de la ciencia política, es solamente a partir del siglo xx cuando podemos encontrar algún intercambio sistemático de investigación a escala internacional, construido a través de estructuras particulares como los encuentros internacionales o las asociaciones entre académicos relevantes. Los periodistas y otras personas vinculadas con el mundo de la prensa organizaron su primer congreso internacional en 1894, seguido de su propia asociación internacional durante la primera mitad del siglo xx (Nordenstreng et al., 2016). Las políticas globales de medios comenzaron a tomar forma en la Liga de Naciones durante los años veinte del S.xx, en un momento en el que la investigación en comunicación no solamente no estaba establecida sino que ya se encontraba dividida en varias tradiciones. Sin embargo, la investigación en comunicación permaneció conspicuamente relegada en sus plataformas y estructuras internacionales hasta el fin de la Segunda Guerra Mundial.

Palabras clave: IAMCR, instituciones internacionales, periodismo, investigación en comunicación.

\begin{abstract}
Histories of the emerging field of mass communication (e.g. Hardt, 2001; Pietilä, 2005) -in continental Europe from the late seventeenth century onward and in the United States from the early nineteenth century onward-lead one to notice how little and how late international institutions have played a role in shaping communication research. Although the roots of the field go back to the classics of sociology and political science, it is only in the twentieth century that we can find any systematic international networking of research, built through particular structures such as international meetings or associations among relevant scholars. Journalists and other "press people" had their first international congress in 1894, followed by their own international association(s) in the first half of the twentieth century (Nordenstreng et al, 2016). Global media policies began to take shape in the League of Nations in the 1920s-at a time when communication research was not only established but already being divided into various traditions. But communication research remained conspicuously remiss on its own international platforms and structures until the end of World War II.
\end{abstract}

Keywords: IAMCR, International institutions, journalism, communication research. 


\section{DISERTACIONES}

ENSAYOS

Anuario electrónico de estudios en Comunicación Social

ISSN: $1856-9536$

Doi: dx.doi.org/10.12804/disertaciones.09.02.2016.03

Volumen 9, Número 2 / julio-diciembre 2016

Versión PDF para imprimir desde

http://revistas.urosario.edu.co/index.php/disertaciones

\section{RESUMO}

As histórias do campo emergente da Comunicação de massas (Hardt, 2001; Pietilä, 2005), na Europa continental desde finais do século xvII em diante e nos Estados Unidos desde começos do século xıx em diante, nos permitem reconhecer o escasso e tardio papel que têm jogado as instituições internacionais em moldar a investigação em comunicação. Apesar de que as raízes do campo remitem aos clássicos da sociologia e da ciência política, é só a partir do século xx quando podemos encontrar algum intercâmbio sistemático de investigação a escala internacional, construído através de estruturas particulares como os encontros internacionais ou as associações entre acadêmicos relevantes. Os jornalistas e outras pessoas vinculadas com o mundo da imprensa organizaram o seu primeiro congresso internacional em 1894, seguido da sua própria associação internacional durante a primeira metade do século xx (Nordenstreng et al., 2016). As políticas globais de meios começaram a tomar forma na Liga de Nações durante os anos vinte do S. xx, em um momento no que a investigação em comunicação permaneceu conspicuamente relegada nas suas plataformas e estruturas internacionais até o fim da Segunda Guerra Mundial.

Palavras-chave: IAMCR, instituições internacionais, jornalismo, investigação em comunicação.

\section{Prehistoria ${ }^{1}$}

La historia de la AIECS/IAMCR remite a los primeros años de la Organización de las Naciones Unidas para la Educación, la Ciencia y la Cultura (unesco), formada como secuela de la Segunda Guerra Mundial. En 1946, unesco propuso un "Instituto Internacional para la Prensa y la Información, diseñado para promover la formación de periodistas y el estudio de los problemas de la prensa a nivel mundial". Esta iniciativa estaba marcada por el idealismo que había inspirado la fundación de la Organización de Naciones Unidas (onu).

En ese momento, los medios masivos incluían principalmente a la prensa, la radio y el cine, ya que la televisión se encontraba todavía en una fase experimental. Debido al rol que jugaron durante la guerra, los medios de masas fueron identificados como un factor importante en las relaciones internacionales. Consecuentemente, una de las primeras conferencias especiales organizadas por la onu en abril de 1948 se consagró a la libertad de información. Sería en esta reunión donde el famoso Artículo 19 sobre Libertad de Expresión e Información fue incorporado a la Declaración Universal de los Derechos Humanos, adoptada por la Asamblea General de la ONU en diciembre del mismo año. Dos fundadores y futuros presidentes de la AIECS -Fernand Terrou y Jacques Bourquin-se implicaron activamente en la preparación del Artículo 19 durante la Conferencia de la onu sobre Libertad de Expresión e Información.

1 Este apartado está basado en Hamelink y Nordenstreng (2007, pp. 6-8), disponible en línea (http://iamcr. org/in-retrospect/preparation) y que contiene fuentes y referencias más detalladas. También se puede consultar: http://iamcr.org/in-retrospect 


\section{DISERTACIONES}

Anuario electrónico de estudios en Comunicación Social

ISSN: 1856-9536

Doi: dx.doi.org/10.12804/disertaciones.09.02.2016.03

Volumen 9, Número 2 / julio-diciembre 2016

Versión PDF para imprimir desde

http://revistas.urosario.edu.co/index.php/disertaciones

Una década pasó, sin embargo, hasta que la Aeıcs fue establecida. Una de las razones detrás de este lento progreso fue el rápido deterioro de las relaciones Este-Oeste y el inicio de la Guerra Fría a finales de los años cuarenta. Asuntos relacionados con el rol de la opinión pública y de los medios generaron preocupación en la política doméstica y se adquirieron una creciente relevancia en las relaciones internacionales. Además, el Instituto Internacional de Prensa (IIP) fue establecido en 1951 como una asociación internacional de editores de periódicos y empresas editoriales en los países occidentales, representando el mundo libre, opuesto al mundo comunista. En ese momento, unEsco se mostró reacia a promover el establecimiento de una nueva asociación de investigación, considerando que el IIP podría cubrir esta necesidad cuando asumió, por ejemplo, un análisis de contenido de los flujos de noticias internacionales.

Sin embargo, las limitaciones de la base geopolítica y temática del IIP resultaron, muy pronto, obvias. unEsCo se dio cuenta de que, además de la libertad de prensa, había otros asuntos importantes en el pujante campo de la comunicación de masas, especialmente relacionados con la educación sobre periodismo, que se beneficiarían de una actividad coordinada a nivel internacional por una organización autónoma. En 1952, unEsco retomó esta cuestión, abriendo dos líneas de acción: establecimiento de centros de formación para periodistas y fundación de una organización internacional para la promoción de investigación científica sobre comunicación masiva.

En este momento, la Secretaría de la unEsco estableció un centro de información (Clearinghouse) dentro de su Departamento de Comunicación de Masas, que recibió el encargo de "recopilar, analizar y difundir información sobre prensa, cine, radio y televisión, destacando su uso para propósitos educativos, culturales y científicos", tal y como se puede leer en el prefacio de su colección Reports and Papers on Mass Communication. Los primeros veinte trabajos de esta colección, publicados entre 1952 y 1956, cubrieron temas relacionados principalmente con el cine, la televisión y la prensa, pero su número de diciembre de 1956 fue titulado Investigación en Comunicación Actual I. Este volumen incluyó un repertorio de los proyectos de investigación en curso y una bibliografía de libros y artículos publicados hasta comienzos de 1955. La lista de investigación en curso incluía cerca de 400 proyectos en catorce países, mientras que la bibliografía enumeraba unas 800 publicaciones en 25 países. Esta impresionante panorámica de investigación fue compilada mediante un cuestionario enviado a 32 instituciones seleccionadas en 19 países. La recogida de datos fue realizada por las cámaras de compensación nacionales radicadas en Francia, Japón y Estados Unidos. Este procesó estimuló el establecimiento de centros de información en otros países, comenzando por la República Federal Alemana e Italia.

El año 1956 fue crucial para el desarrollo bajo los auspicios de la unEsco. En abril, una reunión de expertos en la formación profesional de periodistas fue organizada en las oficinas centrales de la unEsco en París. Esta reunión de cuarenta catedráticos y otros expertos en medios, con la documentación y las resoluciones adoptadas, puso en evidencia la existencia de un campo de investigación dinámico que requería coordinación internacional. Un listado de recursos para la formación profesional de periodistas incluyó un centenar de institutos procedentes solamente de los Estados Unidos, y casi una centena más de unos treinta países más.

En ese punto se organizó un coloquio en Estrasburgo, donde se fundó el Centro para la Educación Superior en Periodismo. Con motivo de esta ocasión, en diciembre de 1956, se formó un grupo preparatorio, denominado Comité Provisional por cuatro investigadores: Fernand Terrou (director del Instituto Francés para la Prensa y presidente de la Asociación Francesa de Ciencias de la Comunicación), Mieczyslav Kafel (director del Instituto de Periodismo en la Universidad de Varsovia), Marcel Stijns (editor-jefe del periódico belga Het Laatste Nieuws y vicepresidente de la Federación Internacional de Periodistas), y David Manning White (catedrático de periodismo 


\section{DISERTACIONES}

Anuario electrónico de estudios en Comunicación Social

ISSN: 1856-9536

Doi: dx.doi.org/10.12804/disertaciones.09.02.2016.03

Volumen 9, Número 2 / julio-diciembre 2016

Versión PDF para imprimir desde

http://revistas.urosario.edu.co/index.php/disertaciones

en la Universidad de Boston y director del Consejo de Investigación de la Asociación Americana para la Educación en Periodismo). Terrou presidió el comité y Jacques Kayser, director de investigación del Instituto Francés para la Prensa sirvió como su secretario ejecutivo.

Las tareas asumidas por la nueva asociación preveían incluido no solamente la promoción general de contactos internacionales dentro del campo sino también funciones específicas de una cámara de compensación, como la producción de bibliografías y listas de instituciones como las publicadas en el inventario de la unEsco. El comité preparó una propuesta de constitución y envió dos circulares a potenciales participantes. Convocó su conferencia inaugural en diciembre, después de que el IIP celebrase su reunión en Asia (Colombo) en noviembre.

En resumen, una vez que la comunicación de masas, como otros campos de actividad socioeconómica, hubo alcanzado un cierto nivel de importancia y especialización en la sociedad, esto condujo a la institucionalización del campo, tanto nacional como internacionalmente. En consecuencia, la AlEcs creció de forma rápida desarrollando el campo de los medios, especialmente en todo lo relacionado con el periodismo, creando su propio abanico de intereses institucionales y una necesidad de formación profesional y, también, de investigación científica. Como Terrou escribió en Etudes de Presse, la publicación periódica del Instituto Francés para la Prensa, en 1956, "la formación profesional de periodistas y la ciencia de la comunicación son la agenda del día", añadiendo que "esto es muy bueno para la libertad de información". Para Terrou, al igual que para Bourquin, AlEcs representó no solamente un proyecto técnico para promover la educación y la investigación, sino también un proyecto ideológico para servir a una causa mayor que pretendía promover la paz y la libertad en un orden internacional.

Por lo que concierne a sus intereses, AIECS se concentró inicial y principalmente en el periodismo y en la comunicación masiva, más que, por ejemplo, en la comunicación oral, que contaba con una extensa tradición académica en los Estados Unidos, o en las telecomunicaciones, que en ese momento permanecían predominantemente como una cuestión técnica. Los actores implicados eran principalmente académicos, con una fuerte presencia de periodistas y personas procedentes de la industria mediática.

El trampolín para la AIEcs fue la combinación de las necesidades de formación con el crecimiento de la investigación en comunicación de masas. A diferencia de otros campos, la emergencia de una asociación científica procedió -en niveles nacionales e internacionales- de acuerdo con las demandas no solamente de la investigación académica, sino también del entrenamiento profesional fuera del ámbito académico. Desde sus comienzos, la investigación en comunicación masiva ha sido inseparable de la formación de comunicadores, especialmente periodistas. Un contraste, por ejemplo, con la ciencia política, que ha jugado un papel muy pequeño en el entrenamiento de políticos. Sin embargo, a pesar de que la educación fue crucial para garantizar que los intereses de investigación consiguiesen reconocimiento internacional, al menos para poner la asociación en marcha, la educación y la investigación requerían permanecer separadas de forma eventual.

Desde el punto de vista geopolítico, la AIECs tuvo una amplia -casi global- base, con instituciones e individuos de todos los continentes afiliados en ella. No hay duda de que la iniciativa de crear la AlEcs estuvo en manos de europeos, particularmente franceses, pero colegas de países como Brasil, Perú, Uruguay, Egipto, Israel, India, Indonesia, Japón, Australia, Estados Unidos y Canadá también estuvieron implicados. La nueva Europa del Este, detrás del denominado telón de acero, fue representada por académicos de Polonia, Checoslovaquia y de la Unión Soviética, haciendo que la configuración de la AIEcs resultase más equilibrada que la del II o la de las dos asociaciones internacionales de periodistas profesionales, la Organización Internacional de Periodistas (oıp, representando 


\section{DISERTACIONES}

ENSAYOS

Anuario electrónico de estudios en Comunicación Socia

ISSN: $1856-9536$

Doi: dx.doi.org/10.12804/disertaciones.09.02.2016.03

Volumen 9, Número 2 / julio-diciembre 2016

Versión PDF para imprimir desde

http://revistas.urosario.edu.co/index.php/disertaciones

principalmente al Este y al Sur) y la Federación Internacional de Periodistas (FIP, representado principalmente al Oeste). Por lo tanto, AIECS no fue un proyecto de la Guerra Fría. Al contrario, fue fundada en suelo ecuménico cruzando las brechas Este-Oeste y Norte-Sur.

\section{Desarrollos²}

La historia de la AIEcs se expande durante seis décadas, con cuatro fases de desarrollo: (1) la fundación, 1957-1964;

(2) un periodo de consolidación, 1964-1972; (3) los años de crecimiento, 1972-1990; y, finalmente, (4) el periodo de los retos, desde 1990. Ha celebrado 29 conferencias bianuales, reflejando su perfil global como lo muestra el siguiente listado:

\section{Conferencias}

1957

1959

1961

1964

1966

1968

1970

1972

1974

1976

1978

1980

1982

1984

1986

1988

1990

1992

1994

1996

1998

2000

2002
París (Francia)

Milán (Italia)

Vevey (Suiza)

Viena (Austria)

Herceg Novi (Yugoslavia)

Pamplona (España)

Konstanz (Alemania, RFA)

Buenos Aires (Argentina)

Leipzig (Alemania, RDA)

Leicester (Reino Unido)

Varsovia (Polonia)

Caracas (Venezuela)

París (Francia)

Praga (Checoslovaquia)

Nueva Delhi (India)

Barcelona (España)

Bled (Yugoslavia)

Guarujá (Brasil)

Seúl (Corea del Sur)

Sydney (Australia)

Glasgow (Escocia)

Singapur

Barcelona (España)

\section{Presidentes}

Fernand Terrou (Francia)

Raymond B. Nixon (Estados Unidos)

Jacques Bourquin (Suiza)

James D. Halloran (Reino Unido)

Cees Hamelink (Holanda)

Hamid Mowlana (Estados Unidos)

Manuel Parés i Maicas (España)

Frank Morgan (Australia)

2 Este apartado está basado en Hamelink and Nordenstreng (2007, pp. 10-19), que se encuentra disponible en los siguientes enlaces: http://iamcr.org/in-retrospect/foundation; http://iamcr.org/in-retrospect/consolidation; http://iamcr.org/in-retrospect/growth 


\section{DISERTACIONES}

Annabelle Sreberny (Reino Unido)

2010

Braga (Portugal)

2012

Durban (Sudáfrica)

Janet Wasko (Estados Unidos)

2014

Hyderabad (India)

\subsection{Fundación, 1957-1964}

La conferencia fundacional se celebró en las oficinas de la unEsco en París en diciembre de 1957. Cincuenta expertos de quince países asistieron para establecer la asociación para la promoción por todo el mundo del avance en la investigación sobre problemas relacionados con la prensa, la radio, la televisión y las películas. La lista de miembros de la asociación incluía unos 200 nombres de institutos, organizaciones educativas y socios individuales. Los educadores en periodismo eran los más numerosos en la lista de socios individuales de educadores y sociólogos.

La primera asamblea general tras la conferencia fundacional fue organizada en Milán, Italia, en 1959. En esta ocasión, la AIECS eligió a su primer presidente estadounidense, Raymond Nixon, mientras que el presidente francés fundacional, Fernand Terrou, se convirtió en secretario general. La mayoría de las personas elegidas como oficiales en 1957 en París fueron reelegidas en Milán, si bien algunos intercambiaron sus posiciones. La asamblea general en Milán modificó la estructura organizacional de la AIECS con una presidencia rotatoria y una secretaría permanente compuesta por una posición de secretario general y otra de secretario general adjunto. A su vez, se establecieron cuatro secciones permanentes para (1) investigación histórica, (2) investigación jurídica y política, (3) investigación psicológica y sociológica e (4) investigación económica y técnica.

Además del trabajo de las secciones, la AIEcs movilizó la atención académica hacia temas concretos mediante coloquios autónomos y sesiones temáticas en sus conferencias bienales. La primera gran empresa temática consistió en un coloquio internacional sobre el secreto profesional de los periodistas, organizado en Estrasburgo en octubre de 1958. A continuación, se realizó un estudio sobre el mismo tema encargado por la unEsco y publicado conjuntamente por la AIECS y por el IIP en 1959. Otro proyecto inicial al que la unESCO invitó a contribuir a la AIECS fue la preparación de una bibliografía seleccionada acerca de la influencia del cine en la infancia. La AIEcs también comenzó a recopilar y publicar bibliografías generales sobre investigación en comunicación masiva -algo que estaba previsto como una de sus tareas principales en la conferencia fundacional. A pesar de que la cosecha de estos inventarios no resultó tan abundante como la acumulada por la Cámara de Compensación de la unesco en 1956, el primer boletín de la AIEcs resultó útil como canal para compartir datos bibliográficos.

La etapa fundacional continuó durante la segunda asamblea general en Vevey, Suiza, en 1961, y la tercera en Viena, Austria, en 1964. El Presidente Nixon promovió el traslado de la secretaría desde París a Amsterdam, con Maarten Rooy como secretario general, Gazzette como la revista científica oficial de la asociación y el Boletín AIECS como su suplemento. En este punto, existía una clara fricción entre franceses y norteamericanos -la unESCO permanecía más próxima a los estadounidenses que a los franceses-, pero formalmente la asociación continuaba funcionando con normalidad. Nixon fue sucedido en Viena por Jacques Bourquin como presidente, abriendo una nueva etapa. 


\section{DISERTACIONES}

ENSAYOS

Anuario electrónico de estudios en Comunicación Social

ISSN: 1856-9536

Doi: dx.doi.org/10.12804/disertaciones.09.02.2016.03

Volumen 9, Número 2 / julio-diciembre 2016

Versión PDF para imprimir desde

\subsection{Consolidación, 1964-1972}

Después de Viena, la secretaría fue trasladada desde Ámsterdam a la oficina del Presidente Bourquin en Lausanne, Suiza, desde donde comenzó a emitir cartas presidenciales. Durante sus ocho años de presidencia, 26 cartas fueron enviadas en inglés y en francés como copias mimeografiadas informando sobre las novedades de la organización. Bourquin las escribió él mismo aunque, como director de la Asociación de la Prensa para la Suiza francófona, él contaba con un asistente personal para las tareas de traducción y de oficina. Las cartas evocan una asociación internacional activa, pero de un modo que recordaba más a una industria artesanal que a un organismo oficial de alcance mundial.

En esta nueva AIEcs radicada en Lausanne, Gazette solamente tuvo una relación nominal con la asociación y su suplemento, el Boletín, fue desapareciendo gradualmente, debido a la falta de financiación. Formalmente, Rooy había sido nombrado como oficial a cargo de las publicaciones, mientras que Terrou fue designado como director de investigación. En la práctica, la función de cámara de compensación de la Alecs se limitó a listados ocasiones de estudios y de publicaciones de sus miembros, distribuidas junto a las cartas presidenciales. Aun así, algunos estudios temáticos se llevaron a la práctica mediante contratos con la unesco, incluyendo una comparación de los estatutos de las compañías de radio y televisión.

La actividad principal de la AIECS durante esta etapa fueron las conferencias bienales y otros simposios; estos contaban con una abundante asistencia y sirvieron como plataformas importantes para los intercambios académicos y políticos. A través de estos encuentros, la membresía aumentó lenta pero firmemente y mantuvo su balance, especialmente entre la Europa del Este y la del Oeste.

La conferencia de 1966, celebrada en Herceg Novi, Yugoslavia, fue un hito en los primeros pasos de la AiEcS. Más de 70 participantes de 17 países de Europa, Asia y los Estados Unidos debatieron, entre otras cosas, sobre los medios masivos y el desarrollo nacional. El tema del desarrollo fue presentado por Gerhard Maletzke, de Alemania, mientras que Lakshman Rao, de India, estuvo presente como representante de la unEsco. Muchos estadounidenses, incluyendo a George Gerbner y a Herbert Schiller, así como Walery Pisarck de Polonia, Yassen Zassoursky de la Unión Soviética y Kaarle Nordenstreng de Finlandia se sumaron a la asociación en esta ocasión.

Nuevas secciones temáticas fueron establecidas en Herceg Novi, para terminología y metodología, para formación profesional, así como para estudios de mercado y publicidad. Esta última sección era lideradas por un estadounidense, Leo Bogart, quien propuso, en una carta dirigida a la asamblea general, una fusión con la Asociación Mundial para la Investigación en Opinión Pública (World Association for Public Opinion Research, waPOR). La asamblea invitó a Bogart a buscar una cooperación estrecha -y eventualmente una fusión- entre su sección y WAPOR. Los participantes previeron una confluencia similar entre la sección de historia y el Consejo Internacional de las Ciencias Históricas (International Council of Historical Sciences). Estas iniciativas no contaron con ningún desarrollo posterior.

En 1968 la AiEcs celebró su asamblea general en Pamplona, España, bajo el régimen de Franco. Paradójicamente, esta sede reemplazó a Oxford, en el Reino Unido, donde los visados no podían ser garantizados para los representantes de los "países comunistas" del Este de Europa. Entonces, en el turbulento 1968, hubo tres banderas rojas en la Universidad de Navarra marcando la presencia de delegados como Emil Dusiska de la principal escuela de periodismo de Alemania del Este, la Universidad Karl Marx en Leipzig. Políticamente, esto era un indicador de las 


\section{DISERTACIONES}

Anuario electrónico de estudios en Comunicación Social

ISSN: 1856-9536

Doi: dx.doi.org/10.12804/disertaciones.09.02.2016.03

Volumen 9, Número 2 / julio-diciembre 2016

Versión PDF para imprimir desde

http://revistas.urosario.edu.co/index.php/disertaciones

tendencias liberales en la sociedad española en el sensible campo de los medios masivos, y sirvió como un estímulo para los elementos radicales entre los estudiantes y el profesorado (Barrera, 2007).

En Pamplona, Zassoursky fue elegido vicepresidente -el primer ruso en posiciones de liderazgo-, aunque su predecesor como Decano de la Facultad de Periodismos en la Moscow State University, Evgeniy Khudyakov, había estado implicado en los procesos preparatorios. Irina Tetelowska, de Polonia, se convirtió en la directora de una nueva sección sobre bibliografía: esta directora del Centro de Investigación sobre Prensa en Cracovia fue la primera mujer en asumir una posición de liderazgo en la asociación. Una nueva sección fue establecida sobre investigación en medios masivos y comprensión internacional, después de un gran simposio internacional sobre este tema celebrado en Ljubljana junto a los miembros yugoslavos de la IAMCR en septiembre de 1968 para resaltar el vigésimo aniversario de la Declaración Universal de los Derechos Humanos. Paradójicamente, esto sucedió justo después de que la Primavera de Praga fuese aplastada.

La asamblea general de 1970 tuvo lugar en Konstanz, Alemania Federal, después de que Moscú fuese tomada en consideración como sede conjunta con la conferencia mundial de historiadores. Aquí la AIEcs adoptó una extensa serie de recomendaciones a las Naciones Unidas y a sus estados miembro sobre varios aspectos relativos a la comunicación de masas, principalmente la libertad de información, la integridad cultural de las naciones, y el uso de satélites. Entre las personas implicadas en emitir las recomendaciones se encontraban Bogdan Osolnik, de Yugoslavia, y Dallas Smythe, de Canadá. Una resolución separada fue aprobada para apoyar a los periodistas en misiones peligrosas y otra en apoyo de los esfuerzos para establecer una Universidad de las Naciones Unidas, con la expresa esperanza de que "la ciencia de la comunicación de masas" recibiese el reconocimiento apropiado a su importancia. Esta conferencia fue la primera a la que asistió James Halloran, quien sería inmediatamente elegido vicepresidente.

Durante este tiempo -desde finales de los años sesenta a comienzos de los setenta- importantes acontecimientos tuvieron lugar en la asociación. Primero, la investigación en comunicación de masas creció por todos los lugares al mismo tiempo que los medios se expandía, especialmente la televisión. Nuevos programas universitarios fueron establecidos y se nombramos comités nacionales para destacar el campo, desembocando en nuevas instituciones como el Centro de Investigación en Comunicación de Masas (Centre for Mass Communication Research) en la Universidad de Leicester, liderado por Halloran. Segundo, la orientación científica y política de la investigación en comunicación se diversificó por la irrupción de académicos críticos, anti-positivistas -entre ellos varios que fueron muy activos en la AIECS, como Smythe desde Canadá y Schiller desde los Estados Unidos- y jóvenes académicos, como Robin Cheesman, Nicholas Garnham, y Armand Mattelart desde la Europa occidental. Tercero, la unESCO asumió un rol mucho más prominente en el campo.

La iniciativa de la unEsco es una historia en sí misma: su explícita orientación hacia la política aproximándose a la generación de académicos críticos y su resonancia global conectándose al Movimiento No Alineado del mundo en desarrollo, con Yugoslavia como uno de sus líderes. La Conferencia General de la unesco aprobó en 1968 una nueva estrategia para promover la investigación y la política de comunicación, incluyendo un estudio sobre el papel y los efectos de la comunicación de masas en la sociedad moderna (unesco, 1970, p. 3). Su Departamento de Comunicación Masiva encargó a Halloran un documento de trabajo sobre medios de comunicación y sociedad para una reunión de expertos celebrada en Montreal en 1969. Halloran también fue consultado sobre quién debía ser invitado y muchos en su lista eran miembros activos de la AIECs, incluyendo a Bourquin, Edelstein, Maletzke, Nordenstreng y Smythe.

\section{4}




\section{DISERTACIONES}

ENSAYOS

Anuario electrónico de estudios en Comunicación Social

ISSN: 1856-9536

Doi: dx.doi.org/10.12804/disertaciones.09.02.2016.03

Volumen 9, Número 2 / julio-diciembre 2016

Versión PDF para imprimir desde

El documento de trabajo de Halloran para Montreal y el informe final de la reunión se publicaron en inglés, francés y español (unEsco, 1970). En conjunto, este encuentro y la publicación suponen un hito en la historia de la investigación en comunicación de masas. Fueron continuados por el panel internacional de consultores en investigación en comunicación de la unesco, que incluyó a Halloran, Nordenstreng, Pisarek, Smythe y otros de la asociación. El panel se reunió por primera vez en abril de 1971 para preparar Propuestas para un Programa Internacional sobre Investigación en Comunicación (Proposals for an International Programme of Communication Research), otro documento histórico (Nordenstreng, 1994).

\subsection{Crecimiento, $1972-1990$}

Buenos Aires, en 1972, fue algo más que la primera conferencia que la AIECS organizaba en América Latina. Marcó una nueva era de cooperación con unEsco, en un momento en el que la investigación en comunicación de masas estaba atravesando lo que sería denominado como un "giro social", la etapa en la que un campo joven toma conciencia de sí mismo y se implica activamente en la política social. El papel de unEsco en Buenos Aires fue crucial, pues asumió los costes de transporte de doce miembros de su panel sobre investigación en comunicación (que organizó su segundo encuentro con motivo de la conferencia). Además, muchas otras personas encontraron medios para viajar hasta Argentina en aquel momento, incluyendo a Dusiska y Schiller.

De acuerdo con la última carta presidencial de Bourquin, la conferencia de Buenos Aires bajo el tema Comunicación y Desarrollo, contó con la asistencia de unos 50 miembros de la IAMCR, además de "una importante participación sudamericana y argentina". Las elecciones resultaron en Halloran como presidente, y Dusiska como secretario general. Nordenstreng y Schiller fueron elegidos vicepresidentes, además de otros cuatro que asumieron estas posiciones en los años previos. Una señal de aquellos tiempos fue el establecimiento de una nueva sección para la investigación de los medios y los países en vías de desarrollo. Alfred Opubor, de Nigeria, fue elegido como director de esta sección, mientras que Annette Suffert, de Francia, fue elegida como directora de otra nueva sección sobre estudios de televisión.

Desde 1973 podemos seguir los avances de la AIEcs en las cartas presidenciales de Halloran, emitidas desde el Centro de Leicester, donde la secretaría fue trasladada de forma efectiva desde la oficina de Bourquin en Lausanne. Las cartas mimeografiadas siguieron el mismo tono familiar establecido por Bourquin, y fueron aumentando su extensión sin cesar, detallando no solamente los acontecimientos y los planes de la organización sino también los encuentros y contactos realizados en su condición de presidente. Estas redes de contactos convirtieron a Jim Halloran en un hombre conocido por centenares de colegas alrededor del mundo y convirtieron a Leicester en el centro de atención del campo, con Peggy Gray como mano derecha del presidente en las cuestiones administrativas.

El secretario general Dusiska, en su oficina de Leipzig, permaneció en un segundo plano, pero cooperó de manera efectiva con Halloran. Dusiska organizó una reunión del comité ejecutivo con un intenso debate acerca del pasado, presente y futuro de la asociación. El comité acogió de forma positiva la propuesta del anfitrión de organizar la siguiente conferencia bienal en Leipzig, empleando para ello los vastos recursos institucionales que Dusiska tenía a su alcance en la Karl Marx University con el respaldo de las autoridades de Alemania del Este. Con 


\section{DISERTACIONES}

ENSAYOS

Anuario electrónico de estudios en Comunicación Social

ISSN: $1856-9536$

Doi: dx.doi.org/10.12804/disertaciones.09.02.2016.03

Volumen 9, Número 2 / julio-diciembre 2016

Versión PDF para imprimir desde

Leipzig, se estableció la tradición de la asociación de albergar las sucesivas conferencias en los hemisferios del Este, del Sur y del Oeste.

La conferencia de Leipzig en 1974 abordó el tema general de Comunicación de masas y Consciencia Social en un Mundo Cambiante, con cuatro subtemas transversales al conjunto del campo: economía, participación, socialización, y naciones en desarrollo. Más de 60 comunicaciones fueron presentadas, y todos ellas junto a los discursos principales fueron impresos por el anfitrión en un libro multilingüe compuesto por dos volúmenes. La conferencia batió la plusmarca de asistencia con 250 delegados procedentes de 31 países. Entre ellos se encontraban, nuevamente, los miembros del panel de la unEsco que se reunieron con anterioridad a la conferencia. El Presidente Halloran alabó a la conferencia en su carta Queridos amigos y colegas de diciembre de 1974:

Para mí, uno de los logros más estimulantes fue encontrarse con nuevas caras en Leipzig. Para una aso-

ciación como la nuestra, es bueno contar con el apoyo continuo y con la lealtad de los viejos amigos, pero

también resulta totalmente esencial atraer y mantener el interés de nuevos y jóvenes investigadores.

En el área de las publicaciones, Halloran informó que la unEsco estaba interesada en financiar la publicación de un Monográfico AEICS que, además de contener artículos y una amplia bibliografía sobre un tema seleccionado que resultase de interés para el campo, incluiría otras dos secciones más. El Monográfico se convirtió en un libro de 130 páginas, publicado con motivo de la siguiente conferencia (Halloran, 1976). Contenía dos artículos importantes sobre medios de comunicación y socialización (escritos por un autor británico y otro soviético), con una amplia bibliografía internacional sobre el tema, compilada por Pisarek. Además, el libro incluyó el perfil de la AeIcs elaborado por Halloran y la presentación realizada por Nordenstreng de un emergente sistema global de centros de información y documentación sobre investigación en comunicación masiva.

La asamblea general en Leipzig mantuvo en sus puestos a los oficiales elegidos en Bueno Aires, pero acordó designar un comité para preparar una propuesta de cara a la renovación de los estatutos de la asociación en un plazo de dos años. La conferencia, según se decidió allí, tendría lugar en Leicester, con un título tomado del Programa Internacional de la unesco: Medios de comunicación de masas y la visión del hombre sobre la sociedad. En el debate de la asamblea general, Gerbner señaló "la interpretación sexista de la visión del hombre" y este recordatorio derivó en la incorporación de Elisabeth Noelle-Neumann como un miembro adicional del comité de preparación de la conferencia, compuesto por Halloran, Dusiska, Nordenstreng y Schiller. Esta fue la primera ocasión en la que las cuestiones de género fueron registradas en las actas de la AEICS.

La conferencia de Leicester se organizó a finales de agosto y principios de septiembre de 1976. Contó con la asistencia de más de 300 académicos desde 40 países, convirtiéndola en el encuentro con mayor participación hasta el momento en la historia de la asociación. El programa de la conferencia estaba dividido en cuatro grandes temas: (1) el estado de la cuestión en la investigación en comunicación, abordado por Lothar Bisky (República Democrática Alemana), George Gerbner (Estados Unidos) y Peter Golding (Reino Unido); (2) estructuras y contextos de producción mediática, tratado por Stuart Hall (Jamaica/Reino Unido), Michael Tracey (Reino Unido) y John Pollock (Estados Unidos); (3) la influencia mediática, abordado por Neville Jayaweera (Sri Lanka/Asociación Mundial para la Comunicación Cristiana, World Association for Christian Communication, wacc), N. Mansurov (Unión Soviética) y Elisabeth Noelle-Neumann (República Federal de Alemania); y (4) medios de comunicación y comprensión internacional, tratado por Luis Beltrán (Colombia/Canadá), Phil Harris (Reino Unido), Al Hester (Estados 


\section{DISERTACIONES}

Anuario electrónico de estudios en Comunicación Social

ISSN: 1856-9536

Doi: dx.doi.org/10.12804/disertaciones.09.02.2016.03

Volumen 9, Número 2 / julio-diciembre 2016

Versión PDF para imprimir desde

http://revistas.urosario.edu.co/index.php/disertaciones

Unidos) y Frank Ugboajah (Nigeria). Los replicantes incluyeron a Jay Blumler, Theodore Glasser, Jn Ekecrantz, Cees Hamelink, Elihu Katz, Ramona Rush y Percy Tannenbaum.

Además de las sesiones plenarias, se organizaron seminarios acerca de temas y de proyectos específicos, incluyendo Indicadores culturales, Mujeres y medios de comunicación, Imperialismo mediático/cultural, Problemas éticos en la comunicación de masas y Comunicación y comunidad (un proyecto con ocho naciones encargado por unesco a la AIECS). La mayoría de las actas registraron temas de gran interés, haciendo de la conferencia en Leicester una plataforma adecuada para intercambios intelectuales. Por supuesto, la conferencia ofreció una panorámica de la investigación sobre comunicación de masas que ningún historiados del campo debería desperdiciar. La conferencia también resultó destacada por la adopción de resoluciones acerca de la necesidad de políticas internacionales de comunicación al servicio del desarrollo democrático, y por el apoyo al derecho universal a la comunicación.

Leicester 1976 fue un hito en la historia organizacional de la AIECS, pues los estatutos fueron revisados, siguiendo las recomendaciones del comité nombrado en Leipzig, y las elecciones completadas de acuerdo con la nueva estructura, según la que el comité ejecutivo habría de ser supervisado por un amplio Consejo Internacional que incluía tanto a miembros ordinarios como a los directores de las secciones. La composición del comité ejecutivo permaneció prácticamente igual, mientras que el Consejo Internacional presentaba un alcance internacional en su composición. La lista de candidatos para las elecciones fue aprobada por la Asamblea General, según lo propuesto por Halloran. Su propuesta fue preparada durante la propia conferencia por Nordenstreng, que intentó alcanzar una representación equilibrada no solamente en términos geopolíticos sino también en los que respectaba a las generaciones y al género. Así, Nordenstreng introdujo en la lista a siete mujeres, incluyendo a Nelly de Camargo (Brasil), Anita Werner (Noruega) y Gertrude Robinson (Canadá). La incorporación de Robinson supuso la salida de Dallas Smythe, quien había sido miembro de la directiva desde 1970.

Después de Leicester, la AIECS publicó otro libro con el apoyo de UNESCO (IAMCR, 1978). Halloran y sus asistentes revisaron los temas principales de la conferencia de Leicester, y relevantes bibliografías fueron compiladas por Pisarek en Cracovia y por especialistas en documentación en otros centros de investigación en comunicación.

La siguiente conferencia se celebró en Varsovia, en septiembre de 1978, con el tema general Medios de comunicación y cultura. La asistencia superó nuevamente la de las reuniones precedentes: casi 500 participantes desde 30 países. La membresía de la asociación ya había alcanzado prácticamente el millar de personas, de más de 50 países. Mientras la afiliación continuaba creciendo, los grupos de interés se comenzaron a organizar mucho mejor. Uno de ellos centrado en una aproximación desde la teoría materialista o marxista a la investigación en comunicación, que se reunió por primera vez, de modo informal, en la conferencia de Leicester y que fue aprobado en la asamblea general de Varsovia, tras un acalorado debate, como una sección denominada Economía Política. La sección sobre formación profesional convocó una sesión especial junto a la estadounidense Asociación para la Educación en Periodismo (Association for Education in Journalism, AEJ) y a la Organización Internacional de Periodistas (International Organization of Journalists, OIJ), con Nordenstreng como presidente desde la conferencia de Leicester.

Como antes, la conferencia también desembocó en un libro apoyado por la unESCO (IAMCR, 1980). Cubrió los cuatro ámbitos temáticos de la conferencia: ideologías, teorías y metodologías de los medios de masas y la cultura; estructura, contenido, e influencia de las culturas nacionales; factores políticos, económicos y tecnológicos de la comunicación a través de las culturas e internacional; y contenidos, valores y efectos de la comunicación a través de las culturas e internacional. Las cuatro revisiones fueron escritas por dos académicos polacos y dos académicos 


\section{DISERTACIONES}

Anuario electrónico de estudios en Comunicación Social

ISSN: 1856-9536

Doi: dx.doi.org/10.12804/disertaciones.09.02.2016.03

Volumen 9, Número 2 / julio-diciembre 2016

Versión PDF para imprimir desde

http://revistas.urosario.edu.co/index.php/disertaciones

británicos, seguidas de una completa bibliografía recopilada con la asistencia de Red Internacional de Centros de Información y Comunicación sobre Investigación en Comunicación (International Network of Information and Communication Centres on Communication Research, COMNET), próxima a la unesco.

La AIECS regresó a América Latina en agosto de 1980, ocho años después de Buenos Aires, a Caracas, Venezuela, para una conferencia titulada Nuevas estructuras de la comunicación internacional. Fue otro evento exitoso, si bien no muchos participantes de otros continentes pudieron asumir los costes del viaje. Entre los presentes, además de la mayoría de los oficiales, se encontraban Nelly de Carmago, de Brasil, y Cees Hamelink, de los Países Bajos: ambos fueron elegidos como vicepresidentes en Caracas. Un punto culminante de la conferencia fue un debate no programado entre Ithiel de Sola Pool y Herbert Schiller sobre el tema de la tecnología de los medios y la ideología. Una consecuencia de la conferencia de Caracas fue un examen crítico del proyecto de informe de la Comisión Internacional para el Estudio de los Problemas de Comunicación. Este proyecto, conocido como el Informe MacBride por el presidente de la comisión, Sean MacBride, acababa de ser emitido y atentamente revisado por varios activistas de la AIECS, derivando en una serie de ensayos (Hamelink, 1980).

En aquel momento, el panel de consultores sobre investigación en comunicación de la unEsco había finalizado su periodo y ya no podía celebrar reuniones paralelas a las conferencias de la AIECs, finalizando así la financiación indirecta de la unESCO. El apoyo de la unESCO a las publicaciones temáticas también era intermitente, una evolución similar a la disminución de su apoyo a comnet. Esto era debido a un cambio en las prioridades del programa de comunicación de la unEsco que, a finales de los setenta, se encontraba crecientemente preocupada por la Comisión MacBride (MacBride et al., 1980). Varios miembros de la AlEcs, incluido el presidente Halloran y el vicepresidente Zassoursky contribuyeron al trabajo de la comisión a través de su secretariado y una serie de artículos de contexto, pero este trabajo pasó por alto a la AIEcs como institución.

Sin embargo, la unEsco sí que contrató a la AIECS para llevar a cabo un importante estudio sobre noticias internacionales (Sreberny-Mohammadi et al., 1985). Además, la sección sobre formación profesional (liderada por Zassoursky, y después por Nordenstreng) movilizó junto a la AEJ, la IOC, la wAcC y asociaciones regionales hermanas en África, Asia y América Latina un proyecto para la promoción de libros de texto sobre educación en periodismo en los países en vías de desarrollo. Este proyecto recibió una importante subvención del nuevo Programa Internacional para el Desarrollo de la Comunicación (International Programme for the Development of Communication, IPDC) de la unesco, gracias a la eficiente labor de presión realizada por Alfred Opubor, quien representó a Nigeria en el comité del IPDC. Más tarde, ya en los noventa, el proyecto continuó con el apoyo de la Agencia Finlandesa para el Desarrollo Internacional (FINNIDA).

Caracas fue seguida por conferencias en París (1982), Praga (1984), Dehli (1986) y Barcelona (1988). En Barcelona, los estatutos de la asociación fueron modificados de nuevo para restringir la presidencia a un único mandato: dos años como presidente-electo, seguidos de cuatro años como presidente, y después dos años más como presidente saliente. Halloran había sido reelegido en tres ocasiones desde Buenos Aires en 1972, convirtiéndolo en el presidente que ha estado más tiempo al frente la asociación, con 18 años (con los dos finales al lado del presidente-electo Cees Hamelink). El iraní-estadounidense Hamid Mowlana se convirtió en vicepresidente, junto a K.E. Eapen de India. Los estatutos revisados no contemplaban una larga lista de vicepresidentes (doce habían sido elegidos en Praga), limitando el número ahora a cinco, y por un máximo de dos después de la conferencia 


\section{DISERTACIONES}

ENSAYOS

Anuario electrónico de estudios en Comunicación Social

ISSN: 1856-9536

Doi: dx.doi.org/10.12804/disertaciones.09.02.2016.03

Volumen 9, Número 2 / julio-diciembre 2016

Versión PDF para imprimir desde

http://revistas.urosario.edu.co/index.php/disertaciones

de Barcelona. El mandato de Halloran finalizó formalmente en Bled en 1990, momento en el que se convirtió en presidente honorario, junto a sus predecesores Bourquin, Nixon y Terrou.

Las conferencias y otras actividades de la IAMCR en los años ochenta continuaron contando con una amplia base y éxito, con Barcelona en 1988 como la más destacada: más de 600 participantes desde 46 países. Las ocho secciones y los 32 grupos de trabajo ad hoc organizaron más de 70 paneles con más de 250 comunicaciones presentadas. Además de este récord de asistencia, Halloran pudo anunciar con orgullo las últimas cifras de membresía: 1850 miembros de 60 países.

Sin embargo, el rápido crecimiento y el dinamismo de la década anterior, en parte estimulado por la ayuda financiera de la unEsCo, ya no estaba presente. La AIECS continuó sus vías habituales de actividad formal, sin introducir un boletín, o su propia revista. Después de que Gerbner se convirtiese en el editor de Journal of Communication, publicado por la Annenberg School for Communication en Philadelphia (Estados Unidos), él sugirió que se convirtiese en una revista de la IAMCR, pero su propuesta fue rechazada por el Consejo Internacional, principalmente por motivos económicos, pero también por las reticencias a ligarse a un único journal, y a uno estadounidense en este caso. Mientras tanto, otras asociaciones en diferentes regiones atrajeron investigadores a sus lugares de origen, incluyendo el Consejo Africano para la Educación en Comunicación (African Council for Communication Education, ACCE), el Centro Asiático para la Información y Comunicación de Medios (Asian Media Information and Communication Centre, Amic) y la Asociación Latinoamericana de Investigadores de la Comunicación (ALAIC).

En 1987 ya se percibía un sentimiento común entre los cuerpos que lideraban la AIECs de que la asociación se aproximaba a un estado de estancamiento y de que era el momento para un relevo generacional, así como para un cambio en la presidencia. Este mensaje fue expuesto a Halloran por primera vez en una carta del presidente de la Asociación Finlandesa para la Investigación en Comunicación de Masas durante un encuentro del comité ejecutivo en Tampere en agosto, sugiriendo dos candidatos para el reemplazo: Hamelink y Mowlana.

Las elecciones en Barcelona 1988 fueron históricas en el sentido de que había una elección abierta para el Consejo Internacional, contando cada posición con un candidato masculino y una candidata femenina, un proceso propuesto por Gerbner. Por supuesto, fue después de 1988 cuando

los miembros femeninos comenzaron a penetrar lo que hasta entonces había sido el 'techo de cristal' de

la dirección de gestión de nuestra organización, gracias en parte a una actitud más igualitaria por parte de nuestros colegas masculinos y la presión de una Red de Mujeres recientemente formada (Robinson, 2002).

Pero el género no solamente abordó la gestión de la AIECS, sino que también atrajo a académicos realizando investigación en medios y género, desembocando en una sección presidida por Madeleine Kleberg, de Suecia. También se formó una nueva sección sobre educación mediática, liderada por Birgitte Tufte, de Dinamarca.

La AIECS fue un testigo cercano al colapso del Comunismo en Europa del Este desde 1989 a 1991, primero en agosto de 1989 en Budapest, donde el secretario general Tamás Szecskö organizó un encuentro del Consejo Internacional durante los días en los que los primeros alemanes del Este escapaban hacia el Oeste a través de su embajada en Budapest - un preludio de la caída del Muro de Berlín en noviembre de 1989. La siguiente conferencia de la AIECS tuvo lugar en Bled, Eslovenia, que en agosto de 1990 se encontraba en un estado de conflicto violento, derivando en su secesión de Yugoslavia. Sin embargo, la ciudad vacacional en el lago Bled acogió una reunión pacífica y profesionalmente eficiente con el tema Avances en Comunicación y Democracia, allanando el camino para el nuevo milenio. 


\section{DISERTACIONES}

ENSAYOS

Anuario electrónico de estudios en Comunicación Social

ISSN: $1856-9536$

Doi: dx.doi.org/10.12804/disertaciones.09.02.2016.03

Volumen 9, Número 2 / julio-diciembre 2016

Versión PDF para imprimir desde

\subsection{Retos ${ }^{3}$}

La lista de conferencias a partir de 1990 es bastante extensa, debido a que se comenzaron a organizar encuentros en los "años intermedios" en sedes que se ofrecían a albergar a la AlEcs entre las reuniones bienales habituales ${ }^{4}$. Comenzando con Estambul en 1991, estos congresos pretendían ser reducidos en sus dimensiones, combinando una reunión del Consejo Internacional con una selección de sesiones plenarias y una serie de reuniones de las secciones temáticas. En la práctica, estos encuentros resultaron muy grandes y ricos en cuanto a su contenido académico. Por ejemplo, Dublín en 1993, con Anthony Giddens como ponente principal, y Oaxaca en 1997 con la celebración del $40^{\circ}$ aniversario de la AIECS, por lo que había poca diferencia entre estos encuentros y la conferencia bienal. Existía, de forma evidente, una demanda para contar con plataformas internacionales y más que suficientes anfitriones entusiastas. Por otra parte, los precios al alza de los viajes internacionales y las mejoras en los servicios de Internet para mantener contactos virtuales redujo en cierto modo el interés espontáneo en utilizar las conferencias de la AIEcs para el establecimiento de redes colaborativas.

Estos avances supusieron un reto que derivó en propuestas variadas: orientar la asociación hacia el trabajo en red a partir de los intereses específicos movilizados por las secciones; restringir al alcance regional los encuentros en años intermedios; y convocar las conferencias principales con una periodicidad menor a la bienal, probablemente cada cuatro o cinco años, del mismo modo que otros congresos científicos a nivel mundial han hecho. Sin embargo, no se alcanzó consenso alguno, y la asociación continuó más o menos como hasta entonces. Para los miembros regulares, las actividades principales fueron organizadas por las secciones, que crecieron en número a pesar de los intentos del comité revisor de secciones de establecer una lógica que evitase la proliferación. Los grupos de trabajo fueron introducidos como una subcategoría de las secciones, desembocando gradualmente en un total de treinta secciones y grupos de trabajo ${ }^{5}$.

Continúa existiendo un reto fundamental que ha perseguido a la AIECS a lo largo de su historia, creado por la tensión entre aproximaciones disciplinares específicas dentro del campo (historia, derecho...) y por un mayor interés general en áreas interdisciplinares de investigación (desarrollo, nuevas tecnologías...). Esto presenta un dilema que no se puede resolver fácilmente a través de acuerdos organizacionales. Esto fue reconocido por Halloran y sus predecesores y, en consecuencia, ellos se mostraron bastante abiertos a diferentes iniciativas y preocupados por alcanzar una verdadera representación institucional. Como Terrou solía decir, no es posible el progreso científico sin una amplia colaboración internacional.

Mantener una asociación mundial -primero por lo que respecta al equilibrio entre Este y Oeste y, después, al equilibrio entre Norte y Sur- ha sido un reto constante en la historia de la AIECS. Ha habido también periodos de fricción entre los diferentes intereses regionales dentro del mundo occidental -regiones hispanohablantes contra regiones angloparlantes, Europa contra América del Norte- pero estos conflictos nunca eclipsaron al interés común por una plataforma global. De hecho, se puede sostener que la geopolítica no ha sido tanto obstáculo

3 Este apartado está basado principalmente en Hamelink y Nordenstreng (2007, pp. 20-21), que se encuentra disponible en: http://iamcr.org/node/3024

4 Para una lista completa de conferencias, véase: http://iamcr.org/history/articles/306-iamcr-history-in-anutshell.html

5 Presentadas en la web de IAmcR: http://iamcr.org/node/2943 


\section{DISERTACIONES}

ENSAYOS

Anuario electrónico de estudios en Comunicación Social

ISSN: 1856-9536

Doi: dx.doi.org/10.12804/disertaciones.09.02.2016.03

Volumen 9, Número 2 / julio-diciembre 2016

Versión PDF para imprimir desde

http://revistas.urosario.edu.co/index.php/disertaciones

como un factor positivo que ha convertido a la AIECS tanto en representativa a nivel internacional como intelectualmente estimulante. Si ha habido obstáculos a lo largo de su historia -como en todas las organizaciones humanas-, estos se encontrarán en las personalidades y su "química", más que en las tradiciones académicas como tales.

La naturaleza cambiante de la comunicación de masas en la era de los nuevos medios y la digitalización también ha presentado muchos retos. Una manifestación de esto fue la decisión de cambiar el nombre de la asociación: Masa fue sustituido por Medios en la asamblea general de Sydney en 1996. La propuesta fue realizada por Wolfgang Kleinwächter, entonces director de la sección jurídica, y fue aprobada sin discusión. El cambio de nombre fue sencillo porque no había necesidad de revisar el acrónimo inglés IAMCR. Además, las versiones del nombre en otros idiomas permanecieron sin modificaciones, pues la palabra "masa" no formaba parte del título en francés, Association Internationale des Études et Recherches sur l'Information et la Communication (AIERI), ni en español, Asociación Internacional de Estudios en Comunicación Social (AIEcs).

El cambio fue un paso natural que reflejaba una tendencia general desde los años noventa para dejar atrás el término masa como un rasgo distintivo del campo y, en su lugar, elevar a los medios como su apelativo central. De acuerdo con ello, muchos programas e instituciones académicas adoptaron medios y comunicación en sus nombres. Por otra parte, comunicación de masas tampoco se había vuelto un término totalmente anacrónico, conservando su estatus como una etiqueta válida para el campo en instituciones individuales y en asociaciones ${ }^{6}$. Cualquiera debe recordar que seis décadas antes el concepto de comunicación de masas era bastante moderno, y en los años cuarenta aparecía escrito incluso en la constitución de la unesco, según la cual la organización estaba encargada de

colaborar en la tarea de avanzar en el conocimiento y comprensión mutua de las gentes, a través de todos los medios de comunicación de masas y recomendar, con tal fin, todos los acuerdos internacionales que sean necesarios para promover el intercambio libre de ideas a través de la palabra y de la imagen (Artículo 2a) ${ }^{7}$.

La asociación ha encarado nuevos retos continuamente, tanto institucional como sustantivamente. El reto institucional se afrontó al expandir relaciones con el sistema de las Naciones Unidas más allá de la unEsco, al incluir al Consejo Económico y Social (Economics and Social Council, Ecosoc) y la Organización Mundial de la Propiedad Intelectual (World Intellectual Property Organization (wIPO), así como a las ONGS, incluyendo a la Federación Internacional de Periodistas (IFJ), que había integrado a la antigua ıos tras la Guerra Fría. Quizás el reto más significativo se presentó por debates particulares en el campo de los derechos humanos en plataformas como la Organización para la Seguridad y la Cooperación en Europa (OSCE) en Helsinki 1992, la Conferencia Mundial de los Derechos Humanos en Viena 1993 y la Cumbre Mundial sobre la Sociedad de la Información (World Summit on the Information Society, wsis) en Ginebra 2003 y en Túnez 2005. Una atención sobre los derechos de comunicación supuso el retorno de la Alecs a sus raíces, cuando sus fundadores participaron en la redacción del Artículo 19 de la Declaración Universal de los Derechos Humanos. Los miembros de la AIEcs ofrecieron un abordaje crítico-analítico, persiguiendo alcanzar un equilibrio entre aquellos con intereses propios en emplear el concepto de derechos humanos como un instrumento para justificar la globalización con fines comerciales, y aquellos que lo hacían con intereses

6 Véase, por ejemplo, la asociación de académicos estadounidenses centrados en el periodismo: http:// www.aejmc.org

7 http://portal.unesco.org/en/ev.php-URL_ID=15244\&URL_DO=DO_TOPIC\&URL_SECTION=201.html 


\section{DISERTACIONES}

ENSAYOS

Anuario electrónico de estudios en Comunicación Social

ISSN: 1856-9536

Doi: dx.doi.org/10.12804/disertaciones.09.02.2016.03

Volumen 9, Número 2 / julio-diciembre 2016

Versión PDF para imprimir desde

democráticos, defendiendo libertades fundamentales persiguiendo mejoras en la sociedad civil y sus medios en el mundo posterior a la Guerra Fría.

Estos retos fueron creados en parte por el alejamiento de la unEsco respecto al llamado Nuevo Orden Mundial de la Información y la Comunicación (Nomic), su abandono del enfoque de la Comisión MacBride, y su aceptación de la solución neoliberal para solucionar los problemas relacionados con la comunicación ${ }^{8}$. Cambios personales en el sector de comunicación de la unEsco se tradujeron en un menor énfasis en la tradición científica que representaban las actividades de la AIEcs. El cambio más obvio sucedió a comienzos de los noventa cuando Alain Modoux, antiguo director de las relaciones de prensa de la unEsco, se convirtió en el director del Sector de Comunicación, sucediendo a Alan Hancock, el experto en investigación y políticas de medios de comunicación. Hasta Hancock, el sector siempre se había mostrado favorable a la investigación en comunicación, tomando a la Alecs como su principal representante. Con Modoux, la AIEcs perdió su estatus especial en la unEsco, que había comenzado a buscar socios entre los propietarios de medios y los círculos de las relaciones públicas. Esto también se vio reflejado en el proceso de reorganización de las ong asociadas con la unEsco, motivo por el cual la AlEcs se encuentra, hoy en día, entre la docena de ong relacionadas con los medios de comunicación, reemplazando su posición previa como una de las tres ong de medios de comunicación y como la única representante en el campo de la investigación (Nordenstreng, 2007).

Como parte de esta reorientación, unESCO ayudó a crear un nuevo cuerpo denominado ORBICOM, la Red Internacional de Cátedras unesco en Comunicación. Fue establecida en 1994 en torno a las denominadas Cátedras unEsco en Comunicación, que fueron dotadas en parte con fondos de la unEsco en varias universidades, sobre todo en países en vías de desarrollo y países exsocialistas. Sin embargo, en la práctica, la mayoría de sus miembros eran representantes académicos e industriales procedentes del Oeste. Formalmente, este nuevo cuerpo no se dirigía contra la AIEcs, pero en la práctica contribuyó a la confusión y división en el campo internacional de la investigación en comunicación.

El paisaje global de las asociaciones en comunicación incrementó su confusión aún más debido al potenciamiento de las asociaciones regionales de investigación, si bien la mayoría de ellas mantienen relaciones amistosas e incluso formalizadas con la IAMCR. La primera de ellas fue Amıc, el Centro Asiático de Información y Comunicación sobre Medios, establecido en 1971, y la más reciente es la Asociación Europea para la Investigación y la Educación en Comunicación (European Communication Research and Education Association, ECREA), fundada en 2005. Además, varias organizaciones internacionales con una naturaleza temática especial han entrado en el campo, entre ellas la Asociación de Estudios Culturales (Association for Cultural Studies, ACs) y la Asociación para Investigadores de Internet (Association for Internet Researchers, AoIR).

Un reto particular fue planteado por la Asociación Internacional de Comunicación (International Communication Association, ICA), que en los años noventa comenzó un proceso dirigido a internacionalizar su membresía más allá de su base predominantemente norteamericana. Al mismo tiempo, Klaus Krippendorff, de la Annenberg School for Communication en la Universidad de Pennsylvania, junto a David Mitchell, de la Universidad de Calgary en Canadá, lanzaron la Federación Internacional de Asociaciones de Comunicación (International Federation of Communication Associations, IFCA), aspirando a establecer una plataforma común para las diferentes asociaciones

8 Para una revisión de estos cambios, véase Mansell y Nordenstreng (2006). 


\section{DISERTACIONES}

ENSAYOS

Anuario electrónico de estudios en Comunicación Social

ISSN: $1856-9536$

Doi: dx.doi.org/10.12804/disertaciones.09.02.2016.03

Volumen 9, Número 2 / julio-diciembre 2016

Versión PDF para imprimir desde

orientadas hacia la investigación que coexisten en el campo. La Alecs se mostró displicente ante esta iniciativa, que era más o menos la misma que su misión original, pero no se opuso abiertamente a ella. Otras asociaciones internacionales y nacionales tampoco se mostraron excesivamente entusiasmadas por sumarse a una plataforma global común para compartir la investigación en el campo. Tras algo de interés inicial, este club voluntario ha permanecido, más o menos, como un tigre de papel.

Con el cambio de milenio, el panorama internacional de las organizaciones de investigación en comunicación y en medios había crecido para resultar abundante y diverso (véase Nordenstreng, 2011), pero ¿qué sugería esto a la AIECS?

Por una parte, podríamos decir que la misión de la AIECs ha sido conseguida exitosamente, ya que el campo se ha expandido no solamente en términos de instituciones nacionales sino también en redes internacionales. Por otra, nos podríamos preguntar hasta qué punto el histórico éxito de esta evolución es atribuible a la AlEcs: ¿ha sucedido a pesar de, más que por causa de la influencia de la AIEcs?

La respuesta de estos autores a esta pregunta es bastante cautelosa, incluso cínica: la AlEcs no puede presentarse como un factor decisivo en la internacionalización del campo. La asociación ha seguido, más que conducido, el desarrollo. Aun así, la AIECS ha jugado un papel vital en movilizar la dimensión internacional del campo, especialmente en las décadas iniciales. Parece improbable que cualquier otro organismo pudiese haber promovido de forma más efectiva los contactos internacionales en un campo tan profundamente enraizado en las condiciones nacionales de la política, la economía y la cultura. Este es un problema de investigación para la sociología de la ciencia, pero en cualquier caso nosotros podemos concluir que una lección global de la historia de la AlEcs es la paradoja -una ironía- del hecho que su importancia relativa parece haber decrecido al mismo tiempo que el campo se ha desarrollado en general, y se ha internacionalizado en particular.

\section{Reflexiones ${ }^{9}$}

La lista de conferencias de la AIECS a lo largo de sus 60 años de historia demuestra el impresionante alcance geográfico de esta asociación. La AIEcs se ha reunido en todos los continentes, y aunque la mayoría de sedes han sido en Europa, la lista incluye también varios países del Sur en vías de desarrollo, comenzando por Argentina en 1972. Los primeros 30 años de esta historia tuvieron lugar bajo las condiciones de la Guerra Fría, cuando la AiEcs jugó un papel especial en facilitar los contactos e intercambios entre Este y Oeste.

Michael Meyen ha publicado recientemente un artículo sobre la AlEcs en el campo de batalla Este-Oeste, exponiendo los intentos de la Alemania del Este para utilizar la asociación como un instrumento en la política de la Guerra Fría (Meyen, 2014). Tal y como puntualizaba Nordenstreng en un comentario al artículo, este aspectos fue solamente un asunto colateral en una amplia instantánea donde la AIECS sirvió como una plataforma constructiva y ecuménica para el contacto académico a través de las brechas políticas y culturales.

Es obvio que la asociación ha proporcionado oportunidades para que muchas ideas emergentes fuesen articuladasy promovidas a escala internacional en el campo. Por ejemplo, el enfoque de género fue introducido en la agenda en

9 Este pasaje está parcialmente basado en el ensayo de los autores IAMCR making history, publicado en IAMCR Newsletter, en diciembre de 2014. Véase: http://iamcr.org/newsletter-201412 


\section{DISERTACIONES}

ENSAYOS

Anuario electrónico de estudios en Comunicación Social

ISSN: 1856-9536

Doi: dx.doi.org/10.12804/disertaciones.09.02.2016.03

Volumen 9, Número 2 / julio-diciembre 2016

Versión PDF para imprimir desde

http://revistas.urosario.edu.co/index.php/disertaciones

Caracas en 1980, desembocando en un grupo de trabajo sobre Roles de género en los medios de comunicación, que en 1990 se convertiría en la sección de Género y Comunicación. La AIECS ha permanecido bastante abierta para permitir que las señales silenciosas en el campo fuesen exploradas y movilizadas, al mismo tiempo que no se permitía a los enfoques predominantes disfrutar de posiciones hegemónicas.

Por otra parte, la fenomenal expansión y diversificación en nuestro campo durante las últimas décadas ha hecho imposible acomodar todos los intereses especiales que conviven en la academia dentro de una asociación internacional. Algunos de ellos han considerado que era más práctico establecer sus propios hogares institucionales fuera de la AIECS (y la ICA), caso de los Investigadores de Internet (AoIR) o del periodismo literario (International Association for Literary Journalism Studies, IALJS). Los aspectos particulares de la comunicación, los abordajes teóricos y culturales, así como las áreas geopolíticas, son demasiado variados para continuar permitiendo una arquitectura armoniosa dentro de una única organización. La investigación en comunicación es, cada vez más, un mundo híbrido.

No obstante, un interés universal no se ha vuelto obsoleto. En paralelo a la diversificación del campo, y con la creciente interconexión de académicos a lo largo y ancho del mundo, la necesidad de plataformas mundiales es cada vez mayor. Por lo tanto, la misión original de la AIEcs todavía resulta muy válida: "proporcionar un foro en el que investigadores y otras personas implicadas en los medios y en la comunicación puedan encontrarse e intercambiar información acerca de su trabajo" (Artículo 2.1.1 de los Estatutos).

Es importante recordar que además de servir como foro, la asociación se ha guiado durante toda su historia por una inspiración procedente de los derechos humanos y por una preocupación por la relevancia social de la investigación académica. Desde su prehistoria, que comienza con la Conferencia de las Naciones Unidas sobre Libertad de Información en 1948 hasta su implicación en la Cumbre Mundial para la Sociedad de la Información a comienzos del siglo xxı, la asociación ha perseguido asuntos como la libertad de expresión, la participación en la vida cultural, el intercambio de conocimiento y el derecho a comunicar. El fallecido presidente Jim Halloran solía urgir a sus colegas académicos que no realizasen investigación de espaldas al mundo y de cara a los libros. Es con este espíritu de buscar la relevancia académica y social como se debe comprender tanto la apertura de la asociación a las conversaciones Este-Oeste como la interacción entre el Sur global y sus asociaciones regionales.

Como consecuencia de su historia, la asociación está obligada a continuar su papel en la academia global, ya que todavía afrontamos grandes cuestiones en los estudios sobre medios y comunicación. Encaramos retos enormes en el área de la reflexión teórica y, en particular, para des-occidentalizar y de-colonizar planteamientos convencionales. Necesitamos encontrar abordajes creativos para el encuentro entre ciencia y política, para la confrontación entre investigador y practicante y para los asuntos relacionados con el género y la etnia. También resulta de crítica importancia que nos involucremos en los estudios sobre el futuro: nuestro significativo pasado nos facilita ilusionantes perspectivas para tratar el futuro de la comunicación pública desde una perspectiva global y local.

\subsection{La asociación como un foro para la reflexión científica}

En las percepciones públicas y políticas de la ciencia, encontrar respuestas a preguntas se presenta de forma prominente como el núcleo principal de la actividad de los científicos, con un mínimo histórico en los programas 


\section{DISERTACIONES}

ENSAYOS

Anuario electrónico de estudios en Comunicación Social

ISSN: 1856-9536

Doi: dx.doi.org/10.12804/disertaciones.09.02.2016.03

Volumen 9, Número 2 / julio-diciembre 2016

Versión PDF para imprimir desde

televisivos de divulgación científica, donde la ciencia se transforma en un juego de solucionar puzles. ¡Qué perversión de nuestra profesión! La ciencia es, ante todo, el arte de hacer preguntas críticas.

Una persona puede pensar acríticamente sobre muchas cosas, pero nadie puede pensar de forma científica en un modo acrítico. ¡Resulta complicado imaginar cómo alguien puede implicarse en la investigación científica sin ser crítico! El pensamiento acrítico puede ser propio de otros discursos en la sociedad, la ciencia solamente puede ser crítica porque su encargo es realizar distinciones y comprometerse en el análisis y en la evaluación. Este ejercicio mental requiere una mente reflexiva. En ciencia no hay espacio para la mente absolutista. El choque esencial que afrontamos en el mundo actual es una colisión de mentalidades que es más básico que las divisiones entre culturas, etnias o religiones: es el choque entre la mente absolutista y la mente reflexiva.

Su mentalidad reflexiva le dice a los científicos que todas las pretensiones de validez -sean estas políticas, morales o religiosas- están abiertas a examen y crítica. La mente reflexiva está deseando comprobar todas las ideas en público, escuchar a aquellos que las critican y está abierta a la necesidad de revisar convicciones previas. El núcleo de la mentalidad reflexiva es la necesidad de realizar preguntas. Desafía, por lo tanto, la tradición filosófica que ha moldeado ampliamente los programas educación del Occidente y en su lugar, inspirándose en Platón, insiste en que la inteligencia debería ser medida a través de la capacidad para responder a las preguntas. La sugerencia de que la ciencia equivale a encontrar respuestas inteligentes a las preguntas es fatal para el desarrollo de un pensamiento creativo y crítico. En la mentalidad reflexiva, la inteligencia se mide por su capacidad para formular preguntas. La pedagogía reflexiva permite a los niños tener preguntas e incertidumbres, y así desarrollar sus propias visiones y sus propios sueños sobre el mundo. Habilitar el arte de hacer preguntas implica inevitablemente que la incertidumbre cuente con su propio espacio. Resulta muy prometedor para el desarrollo científico que los cosmólogos actuales estén dispuestos a aceptar que el universo se compone de alrededor de un $96 \%$ de energía oscura y de materia que no comprendemos o que no podemos explicar. En una línea similar, un paso importante para la investigación avanzada en genética que se mire a sí misma como principalmente 'guiada por la ignorancia'.

La mente reflexiva desafía nociones como las de verdad absoluta o de completa certidumbre. Evita las fundamentaciones sólidas, los anclajes y las creencias fijas de que debería existir un conocimiento indubitable para evitar que el mundo se pierda de forma irremediable. La reflexividad reconoce que Immanuel Kant liberó a la mente humana para la exploración especulativa y del anhelo cartesiano de conocimiento cierto. La ciencia no trata sobre la prueba definitiva, sino sobre aproximaciones temporales a la realidad que podrán ser reemplazadas por mejores aproximaciones. Para este ejercicio reflexivo de naturaleza crítica, la ciencia necesita plataformas cuyas características esenciales son:

- Libertad discursiva, que consiste en la capacidad de estar en acuerdo o en desacuerdo con las posiciones de otras personas sobre la base de razones que nosotros podemos evaluar y cuya validez podemos aceptar o rechazar con libertad;

- Respeto a esta libertad discursiva que implica el reconocimiento de la dignidad de la agencia del otro, al aceptar su autonomía para aceptar o rechazar afirmaciones que nosotros consideramos esenciales para nuestras identidades;

- Comportamiento cooperativo, confianza, diversidad y movilidad: los elementos clave -según Charles Darwinpara la supervivencia de las especies; 


\section{DISERTACIONES}

ENSAYOS

Anuario electrónico de estudios en Comunicación Social

ISSN: 1856-9536

Doi: dx.doi.org/10.12804/disertaciones.09.02.2016.03

Volumen 9, Número 2 / julio-diciembre 2016

Versión PDF para imprimir desde

- Creatividad en el trato con la multiplicidad de identidades humanas;

- Capacidad para improvisar al enfrentarse a la incertidumbre;

- Aceptación de la provisionalidad de todas las soluciones a los problemas complejos de la adaptación humana a unos acontecimientos que son siempre más rápidos;

La plataforma que requiere la ciencia necesita las características de una banda de jazz, en la que sus miembros no hacen música contra ellos sino con ellos, aprenden de sus propios errores sin ser castigados, encuentran la clave acertada para balancearse juntos y pasan tiempo probando nuevas ideas.

Sin ninguna declaración de canonización, hemos experimentado desde comienzos de los años setenta del siglo XX que la asociación, entre otras plataformas, se aproxima mucho a esta plataforma ideal.

Inevitablemente, hemos tenido altibajos en la calidad de nuestra reflexión crítica y el espíritu colegial ha dejado mucho que desear a menudo. La banda ha desafinado alguna vez. Pero también ha tenido la buena suerte de contar con muchos académicos en nuestro seno que han conseguido marcar la diferencia en nuestra comprensión del papel que juegan los procesos comunicativos en mundo problemático.

El reto clave para los estudios sobre comunicación y cultura remite a si este campo de investigación científica puede transitar hacia la aventura de cuestiones acerca de la ética y las perspectivas de la vida (humana) y, por lo tanto, aceptar responsabilidad para el ecosistema social que investiga.

Con este espíritu debemos continuar proporcionando una contribución esencial para la ciencia de la comunicación y a la supervivencia de las especies comunicativas en el planeta.

Somos todavía jóvenes y nuestro viaje desde 1957 nos promete un futuro brillante. Cuando Sócrates se presentó ante sus jueces en Atenas, él argumentó que la crítica comienza con el ejercicio de la auto-reflexión crítica. Él advirtió al tribunal que "la vida no examinada no es digna de ser vivida". La AIECS debe seguir proporcionando un foro para el tipo de auto-examen y el pensamiento reflexivo que hace que la vida académica sea digna de ser vivida.

\section{Referencias}

1. Barrera, C. (2007). The IAMCR International Conference at Navarra (Spain) in April 1968: A Little History. En Conferencia de IAmCR, París, 23-25 de julio.

2. Halloran, J. (Ed.) (1976). Mass Media and Socialization: International Bibliography and Different Perspectives. Leeds: IAMCR.

3. Hamelink, C. (Ed.) (1980). Communication in the Eighties: A Reader on the "MacBride Report. Rome: IDoc (Reimpreso en Whitney, C.; Wartella, E. y Windahl, S. (Eds.). Mass Communication Yearbook, 3, pp. 236-87. Beverly Hills, CA: Sage, 1982.)

4. Hamelink, C. (2008). International Association for Media and Communication Research (IAMCR). En Donsbach, W. (Ed.), International Encyclopedia of Communication. Malden, MA: Blackwell Publishing.

5. Hamelink, C. \& Nordenstreng, K. (2007). IAMCR in Retrospect 1957-2007, L'AIERI en retrospective 1957-2007. París: Maison des Sciences de l'Homme, Paris Nord Université Paris 13. Recuperado de: http://iamcr.org/ in-retrospect 


\section{DISERTACIONES}

ENSAYOS

Anuario electrónico de estudios en Comunicación Social

ISSN: 1856-9536

Doi: dx.doi.org/10.12804/disertaciones.09.02.2016.03

Volumen 9, Número 2 / julio-diciembre 2016

Versión PDF para imprimir desde

http://revistas.urosario.edu.co/index.php/disertaciones

6. Hardt, H. (2001). Social Theories of the Press: Constituents of Communication Research, 1840s to 1920s (2a Ed.). Lanham, MD: Rowman \& Littlefield.

7. IAMCR. (1978). Mass Media and Man's View of Society: A Conference Report and International Bibliography. Leicester: IAMCR.

8. IAMCR. (1980). Mass Media and National Cultures: A Conference Report and International Bibliography. Leicester: IAMCR.

9. MacBride, S. et al. (1980). Many Voices, One World: Towards a New More Just and More Efficient World Information and Communication Order. París: UNESCO.

10. Mansell, R. \& Nordenstreng, K. (2006). Great Media and Communication Debates: WSIS and the MacBride Report. Information Technologies and International Development, 3(4), pp. 15-26. Recuperado de: http:// itidjournal.org/index.php/itid/article/view/235/105

11. Meyen, M. (2014). IAMCR on the East-West Battlefield. International Journal of Communication, 8, pp. 20712089. Recuperado de: http://ijoc.org/index.php/ijoc/article/view/2443

12. Nordenstreng, K. (1994). The UNESCO Expert Panel with the Benefit of Hindsight. En Hamelink, C. \& Linne, O. (Eds.). Mass Communication Research: On Problems and Policies, pp. 3-19. Norwood, NJ: Ablex.

13. Nordenstreng, K. (2007). IAMCR as NGO. IAMCR Newsletter, 17(1), p. 2.

14. Nordenstreng, K. (2011). Lost in Abundance? Reflections on Disciplinarity. En Zelizer, B. (Ed.). Making the University Matter, pp. 194-205. New York: Routledge.

15. Nordenstreng, K.; Björk, U.; Beyersdorf, F.; Høyer, S. \& Lauk, E. (2016). A History of the International Movement of Journalists: Professionalism Versus Politics. Houndmills: Palgrave Macmillan.

16. Pietilä, V. (2005). On the Highway of Mass Communication Studies. Cresskill, NJ: Hampton Press.

17. Robinson, G. (2002). IAMCR Then and Now: Lessons from Gender Research. En Conferencia IAMCR, Barcelona, 21-25 de julio.

18. Sreberny-Mohammadi, A.; Nordenstreng, K.; Stevenson, R. \& Ugboajah, F. (Eds.). (1985). Foreign News in the Media: International Reporting in 32 Countries. París: UNESCO (Reports and Papers on Mass Communication, 93).

19. UNESCO. (1956). Current Mass Communication Research - I. París: UNESCO (Reports and Papers on Mass Communication, 21).

20. UNESCO. (1970). Mass Media in Society: The Need of Research. París: UNESCO (Reports and Papers on Mass Communication, 59). 